\title{
Psycological Effect of Slow Breathing
}

\author{
Manendra Kumar More ${ }^{1}$, Komal Meshram² and Ragini Patil ${ }^{3}$ \\ ${ }^{1}$ First Year MBBS Student Datta Meghe Medical College, Shalinitai \\ Meghe Hospital and Research Centre, Nagpur, India \\ ${ }^{2}$ Department of Physiology Datta Meghe Medical College, Shalinitai \\ Meghe Hospital and Research Centre, Nagpur, India \\ ${ }^{3}$ Department. of Psychiatry Jawaharlal Nehru Medica College Datta Meghe Institute \\ of Medical Sciences, Sawangi (Meghe), Wardha \\ Corresponding author email: meshram.komal3@gmail.com
}

\begin{abstract}
Breathing is a necessary for human. It does so much effect on us in physiological and psychological way, we breath in many ways one is normal silent breath and other is breathing exercise. In our daily life, breathing exercise also of two types fast pace and slow pace breathing, in this review article. I had focused on the psychological effect and some physiological effect by slow pace breathing, how slow breathing can help us to overcome little psychological problems in our daily life and somewhat it also helps to prevent major psychological and physiological diseases. Psycological Effect Of Slow Breathing From the review of the articles done by me I would like to conclude with my review that slow breathing exercise is a best way to avoid the psychological disorder we can face today due to the stress and anxiety occurred by work, relation, and emotional disturbance and in some studies, it showed that it can be used as a primary treatment for psychological as well physiological disturbances.
\end{abstract}

KEY WORDS: BREATHING TECHNIQUE, SYSTOLIC BLOOD PRESSURE, DYSTOLIC BLOOD PRESSURE, SLOW BREATHING, INCREASE, ACCORDING TO, BLOOD PRESSURE.

\section{INTRODUCTION}

Breathing is a necessary for human. It does so much effect on us in physiological and psychological way, we breath in many ways one is normal silent breath and other is breathing exercise In our daily life, breathing exercise also of two types fast pace and slow pace breathing, in this review article. I had focused on the psychological effect and some physiological effect by slow pace breathing, how slow breathing can help us to overcome

Biosc Biotech Res Comm P-ISSN: 0974-6455 E-ISSN: 2321-4007

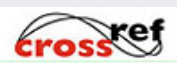

Identifiers and Pagination

Year: 2021 Vol: 14 No (6) Special Issue

Pages: 253-256

This is an open access article under Creative

Commons License Attribn 4.0 Intl (CC-BY).

DOI: http://dx.doi.org/10.21786/bbrc/14.6.52 little psychological problems in our daily life and somewhat it also helps to prevent major psychological and physiological diseases.

\section{METHADOLOGY}

In this review article I had got results of 14331 out of which 25 articles were reviewed and out of these 13 articles were excluded and 12 were included in this review article,I had also found some case study related to it in this article. The main psychological effect of breathing exercise according to the (Jerath et al. 2015) ithypothezise that reversing haomeostatic alternation by breathing or meditation may be better than effecting neurotransmitter with medicines better way to overcome stress, anxiety and depression. According to them BT should be first treatment for stress, anxiety, depression and emotional disorders. (Cramer et al. 2016)BT and yoga postures can help in control over the hypertension acc.

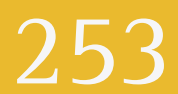


to them hypertension is a one of cause of mortality.Yoga and BT combinations of both can be a alternative for treatment of hypertension yoga effects hypertension by decreasing SBP by $10 \mathrm{mmhg}$ and DBP by $8 \mathrm{mmhg}$. How does effect decreasing in SBP and DBP they told that BT and yoga. Parasympathetic activity and decreases sympathetic activity by incs. GABA activity which counter effects the sympathetic nervous system which effects the hypertension BT with yoga may be safe and effective method to manage hypertension than use of medicines initially.

(Zaccaro et al.2018) shows that pain bais respiration by increasing its flow frequency and volume. But slow paced breathing can be help to reduce the physiological pain but for evidences of this physiological mechanism is lacking. (Qi et al. 2020) One of the research tells us that slow pace breathing can help us to change our life According to them slow pace breathing surfacesANS \& CNS which promote inc. in HRV and RS parallel by CNS. Through MRI study intensify inc. activity of cortical (prefrontal motor, parietal cortices) \&t subcortical. Above given causes changes that incs. Comfort, relaxation, kindliness \&t alertness which reduces the symptoms of arousal, anxiety, depression, anger and confusion. Slow breathing strengthen autonomic,cerebral and psychological flexile in framework of mutual interaction and founded a link betweenparasympatheticactivity, CNS activity allied to emotional control.

This research hypothesis two tools to explain psychophysiological changes by SB:

1. Voluntary regulation of internal bodily state

2. Role of mechanoreceptors within the nasal vault in translating slow breathing in a modulation of olfactory bulb activity, which in turn tunes the activity of the entire cortical mantle.

(Hopper et al. 2019) We often made a mistake of thinking breathing exercises and meditation are of same thing but when I saw this research it told that they seems to be same but are different In some aspects lets see whatresearch.

\section{Yoga Breathing Increases the Quality of our life by}

1. Reducing stress

2. Reducing Pain

3. Reducing Anxiety

4. Reducing Depression

5. Reducing Fatigue

6. Improving sleep and emotions

7. Improves readiness to do tasks

(Hopper et al. 2019) It effects the both ANS and CNS.

\section{Whereas meditation helps to}

1. Serenity or calmness of mind

2. Relaxation

Therefore, breathing may help in achieving mindfulness is much greater than that of meditation does. (Brown et al. 2013) So, promote work intensions breathing practice yoga is much better to focused than meditation practice yoga. we have seen many research of BT related to yoga now I had also found a short research related to BT i.e. taichi it is a Chinese martial art technique which consist of control over our breath it helps us on diff. psychological comfort ofdepression, anxiety, stress management and exercise self-efficiency.

There is also another BT according to (Chaddha et al. 2019) is diaphragmatic breathing which reduces physiological and psychological stress by

1. improving respiratory rate

2. improvement in SBP \& DBP

BT is also help in primary treatment of psychiatric and stress related medical condition. Nuerophysiological studies may explain how BT normalizes stress response, emotional regulation, autonomic Etnueroendocrine system function. BT reveal decrease indication of stress, anxiety, insomnia, post traumatic stress disorder and ADHD. (Brown et al. 2005) According to it BT will be helpful In mental health treatment. BT had also not shown effect on psychological effect but also have some physiological effect on our body too. Some researches also showed that slow breathing help to decrease BP.

(Dhaniwala et al. 2020) Research done by Ashish Chaddha et al. had done a cross over study subjects with hypertension, pre-hypertension Eton antihypertensive medication. Method - SB for 10 breaths/min for more or equal to $5 \mathrm{~min}$. for more or equal to 3 days/week for more than 4 weeks or 1 month. Results - SB lessen the SBP by $-5.62 \mathrm{mmhg}[-7.86$ to -3.30$]$ \&t DBP by $-2.91 \mathrm{mmhg}[-4.28$ to- 1.60]. (Mohamed et al. 2018) Therefore SB decreases $\mathrm{BP}$ and this method can be helped to use as foremost therapy for reducing hypertension and pre-hypertension. As I told earlier in my researchthat combination of yoga and slow BT makes psychological effect in another article reviewed by me of Richard P Brown ${ }^{10}$ told about the Sudarshan Kriya i.e. it helps to reduce the treatment cost of psychological diseases occur by stress, anxiety, depression and PTSD (post traumatic stress disorder).

So far now I would like to end my review research by two case study done by the researchers so let's see what are they. (Mohamed et al. 2018) First case study is of Dr. Sarika Chaudhary is done on football players of a university they were divided into 2 groups of people one group was made to practice Buteyko Breathing Technique (BBT).

And other was not. (Naik et al. 2018) Buteyko Breathing Technique (BBT) it is a type of BT founded by Dr. Konstantin Buteyko this technique comprises of two steps. 
1. In this the players were said to inspiration for 4 sec and then expiration for $3 \mathrm{sec}$ and just after expiration they should hold the breath up-to when they need to have breath and count up-to that time this step is called as control pause.

2. Now the second step is shallow breathing in this the participants were said to put finger just at the top of the lip and to inhale and exhale and feel the warm of breath and they were instructed to try to slow down the warmer breath gradually

3. Thisstep composes of both the above steps first control pause then shallow breathing for 4 min then control pause

The players were made to practice this daily morning and evening time.

And results were as given.

This case study showed that Buteyko Breathing Technique (BBT)

Physiological effects -

1. decrease in heart rate by $5.4 \%$ in group A whereas nothing seems in group B

2. decrease In BP - decrease of SBP by $4.26 \%$ and DBP by $5 \%$ in group $A$ whereas no change in group $B$

3. increase in vo2 max by $15.54 \%$ in group $\mathrm{A}$ and no change in group $B$

Psychological effects

1. Decrease in anxiety by $12.4 \%$ in group A and $4.5 \%$ in group B.

So I conclude that this BT can help to decrease anxiety of a normal person or a sports person by $12.4 \%$ and it also effects o physiology of human body Another case study related to BT was done by G K Pal et al. in this study they have volunteered some medical students and divided in two groups SB(slow breathing) group and $\mathrm{FB}$ (fast breathing) group. They made these students with SB to practice slow breathing exercises for 3 months twice a day and FB group also to practice fast breathing exercises same duration

Results - there were some changes in the autonomic functions of students.

In SB group

1. i.e. increment of parasympathetic activities

2. lessen in sympathetic activities

No changes were observed in FB group

Conclusion from this case study - I can conclude that SB can help -

1. Decrease in stress and strain.

2. Can help in improvement of autonomic and pulmonary functions in asthma patients

1. Can help in treatment of anxiety disorder

2. Decrease in HR and BP by improving vagal tone and decrease in sympathetic discharge.

\section{CONCLUSION}

From the review of the articles done by me I would like to conclude with my review that slow breathing exercise is a best way to avoid the psychological disorder we can face today due to the stress and anxiety occurred by work, relation, and emotional disturbance and in some studies it showed that it can be used as a primary treatment for psychological as well physiological disturbances.acc.to some philosophy SB helps us to connect ourselves to god or infinite power. At last I would like to say that we should practice SB everyday for least 20 min a day with perseverance for months without hoping of instant results.

\section{REFERENCES}

Brown, R.P. and Gerbarg, P.L., 2005. Sudarshan Kriya yogic breathing in the treatment of stress, anxiety, and depression: part I-neurophysiologic model. Journal of Alternative \& Complementary Medicine, 11(1), pp.189201.

Brown, R.P., Gerbarg, P.L. and Muench, F., 2013. Breathing practices for treatment of psychiatric and stress-related medical conditions. Psychiatric Clinics, 36(1), pp.121-140.

Chaddha, A., Modaff, D., Hooper-Lane, C. and Feldstein, D.A., 2019. Device and non-device-guided slow breathing to reduce blood pressure: A systematic review and meta-analysis. Complementary therapies in medicine, 45, pp.179-184.

Cramer, H., 2016. The efficacy and safety of yoga in managing hypertension. Experimental and Clinical Endocrinology \& Diabetes, 124(02), pp.65-70.

Dhaniwala, N.K.S., Dasari, V. and Dhaniwala, M.N., 2020. Pranayama and Breathing Exercises-Types and Its Role in Disease Prevention \& Rehabilitation. Journal of Evolution of Medical and Dental Sciences, 9(44), pp.3325-3331.

Hopper, S.I., Murray, S.L., Ferrara, L.R. and Singleton, J.K., 2019. Effectiveness of diaphragmatic breathing for reducing physiological and psychological stress in adults: a quantitative systematic review. JBI Evidence Synthesis, 17(9), pp.1855-1876.

Jerath, R., Crawford, M.W., Barnes, V.A. and Harden, K., 2015. Self-regulation of breathing as a primary treatment for anxiety. Applied psychophysiology and biofeedback, 40(2), pp.107-115.

Latchoumi, T.P., Ezhilarasi, T.P. and Balamurugan, K., 2019. Bio-inspired weighed quantum particle swarm optimization and smooth support vector machine ensembles for identification of abnormalities in medical data. SN Applied Sciences, 1(10), pp.1-10. 
Mohamed, E.M.H., ELmetwaly, A.A.M. and Ibrahim, A.M., 2018. Buteyko Breathing Technique: A Golden Cure for Asthma. American Journal of Nursing, 6(6), pp.616-624.

Naik, G.S., Gaur, G.S. and Pal, G.K., 2018. Effect of modified slow breathing exercise on perceived stress and basal cardiovascular parameters. International journal of yoga, 11(1), p.53.
Qi, X., Tong, J., Chen, S., He, Z. and Zhu, X., 2020. Comparing the Psychological Effects of editation-and Breathing-Focused Yoga Practice in Undergraduate Students. Frontiers in psychology, 11, p.3036.

Zaccaro, A., Piarulli, A., Laurino, M., Garbella, E., Menicucci, D., Neri, B. and Gemignani, A., 2018. How breath-control can change your life: a systematic review on psycho-physiological correlates of slow breathing. Frontiers in human neuroscience, 12, p.353. 